\title{
Caractérisation De L'aquifère Superficiel Du Bassin Sédimentaire De Kandi (Nord-Est Bénin, Afrique Ouest): Nature Et Structure Du Reservoir, Hydrodynamique
}

\author{
Gaya Salifou Orou Pete Alou \\ Houégnon Géraud Vinel Gbewezoun \\ Aoulatou Alassane Zakari
}

Laboratoire d'Hydrologie Appliquée, Institut National de l’Eau, Université d’Abomey-Calavi, Bénin

Chaire Internationale en Physique Mathématique et Applications (CIPMAChaire UNESCO), Faculté des Sciences et Techniques / Université

d'Abomey-Calavi, Bénin

\section{Abdoukarim Alassane}

Agnidé Emmanuel Lawin

\section{Daouda Mama \\ Moussa Boukari}

Laboratoire d'Hydrologie Appliquée, Institut National de l'Eau, Université d’Abomey-Calavi, Bénin

Doi:10.19044/esj.2021.v17n3p79

Submitted: 28 September 2020

Accepted: 23 December 2020

Published: 31 January 2021
Copyright 2021 Author(s)

Under Creative Commons BY-NC-ND

4.0 OPEN ACCESS

Cite As:

Orou Pete Alou, G. S., Gbewezoun, H. G. V., Alassane Zakari, A., Alassane, A., Lawin, A. E., Mama, D., \& Boukari, M. (2021). Caractérisation De L'aquifère Superficiel Du Bassin Sédimentaire De Kandi (Nord-Est Bénin, Afrique Ouest): Nature Et Structure Du Reservoir, Hydrodynamique. European Scientific Journal, ESJ, 17(3), 79.

https://doi.org/10.19044/esj.2021.v17n3p79

\section{Résumé}

Cet article aborde la caractérisation du bassin sédimentaire de Kandi, notamment sa structure hydrogéologique et son fonctionnement hydrodynamique qui sont encore mal connus, précisément dans sa portion superficielle captée par des forages d'exploitation et directement concernée par l'impact de la variabilité et des changements climatiques. L'analyse de la structure du système aquifère du bassin a été menée par corrélation géologique et hydrogéologique de logs de forages existants. L'étude hydrodynamique a été conduite en considérant les données de pompage d'essai existantes et deux 
campagnes de mesures piézométriques (août 2016 pour les hautes eaux, puis avril 2017 pour les basses eaux). Les résultats confirment la nature essentiellement gréseuse et conglomératique de la lithologie des deux couches captées par les forages hydrauliques, à savoir, selon les secteurs, la formation continentale de Wèrè (Cambro-Ordovicien) et la formation marine de Kandi (Ordovicien supérieur à Silurien inférieur). Les nappes phréatiques de ces deux formations plus ou moins perméables et directement superposées l'une sur l'autre, sont en interconnexion hydraulique pour former une seule et même nappe d'eau souterraine. Les valeurs des paramètres hydrodynamiques calculées montrent que la transmissivité du sytsème aquifère est de l'ordre de 3,08E-02 à 2,99E-05 m²/s, et la perméabilité de l'ordre de 1,30E-03 et 3,37E$06 \mathrm{~m} / \mathrm{s}$. L'écoulement de l'eau souterraine s'effectue globalement du Sud vers le Nord, conformément au pendage régional des couches et à la tectonique.

Mots-clés: Bassin Sédimentaire De Kandi, Stratigraphie, Nappe Phréatique, Profondeur De La Nappe, Piézométrie

\title{
Characterization Of The Shallow Aquifer Of The Sedimentary Kandi Basin (North-East Benin, West Africa): Nature And Structure Of The Reservoir, Hydrodynamic
}

\author{
Gaya Salifou Orou Pete Alou \\ Houégnon Géraud Vinel Gbewezoun \\ Aoulatou Alassane Zakari
}

Laboratoire d'Hydrologie Appliquée, Institut National de l'Eau, Université d’Abomey-Calavi, Bénin

Chaire Internationale en Physique Mathématique et Applications (CIPMAChaire UNESCO), Faculté des Sciences et Techniques / Université d'Abomey-Calavi, Bénin

Abdoukarim Alassane

Agnidé Emmanuel Lawin

Daouda Mama

Moussa Boukari

Laboratoire d'Hydrologie Appliquée, Institut National de l'Eau, Université d’Abomey-Calavi, Bénin

\begin{abstract}
This article deals with the characterization of sedimentary Kandi basin, in particular its hydrogeological structure and hydrodynamic functioning, which are still poorly known, precisely in its superficial part captured by
\end{abstract}


exploitation boreholes and directly concerned by the impact of climate variability and change. The analysis of the structure of the basin's aquifer system was carried out by geological and hydrogeological correlation of existing borehole logs. The hydrodynamic study was carried out by considering existing test pumping data and two piezometric measurement campaigns (August 2016 for high waters, then April 2017 for low waters). The results confirm the essentially sandstone and conglomeratic nature of the lithology of the two layers captured by the hydraulic boreholes, namely, depending on the sector, the continental Wèrè formation (CambroOrdovician) and the marine Kandi formation (Upper Ordovician to Lower Silurian). The water tables of these two more or less permeable formations, which are directly superimposed on each other, are hydraulically interconnected to form a single groundwater table. The values of hydrodynamic parameters calculated show that transmissivity of the aquifer system ranges from $3.08 \mathrm{E}-02$ to $2.99 \mathrm{E}-05 \mathrm{~m} / \mathrm{s}$, and permeability ranges from $1.30 \mathrm{E}-03$ to $3.37 \mathrm{E}-06 \mathrm{~m} / \mathrm{s}$. Groundwater flow is generally from South to North, in accordance with the regional dip of the layers and tectonic.

Keywords: Sedimentary Kandi Basin, Stratigraphy, Water-Table Aquifer, Aquifer Depth, Piezometry

\section{Introduction}

Le Bénin utilise essentiellement les eaux souterraines pour l'approvisionnement en eau potable (AEP) de sa population, car elles sont en général de meilleure qualité, comparativement aux eaux de surface. (Gbewezoun, 2013). Cependant, la faible connaissance des caractéristiques hydrogéologiques des terrains est de nature à hypothéquer lourdement la préservation de ces eaux pour les générations futures (Boukari, 2007).

Le bassin sédimentaire de Kandi (BSK) est l'une des quatre provinces hydrogéologiques identifiées au Bénin par CEFIGRE (1984) et Boukari (2007). Peu de travaux hydrogéologiques lui ont été consacrés, bien qu'il constitue la principale source d'approvisionnement en eau potable (AEP) des populations du Nord-Est du pays (environ 500000 habitants, INSAE 2016), c'est-à-dire, entièrement ou partiellement, celles des Communes de Kandi, Malanville, Ségbana, Karimama, Gogounou et Kalalé (Boukari, 2012). Or, avec la croissance démographique dans ces Communes, le système aquifère à nappe phréatique du BSK est de plus en plus sollicité pour les besoins essentiellement domestiques, agricoles et d'élevage. D’où la nécessité d'approfondir sa connaissance, afin de contribuer à mettre en place une stratégie de gestion durable et rationnelle de ses ressources en eau et de garantir la protection de celles-ci (Gbewezoun, 2017). C'est dire que cette étude s'inscrit opportunément dans le cadre du sixième Objectif de 
Développement Durable (ODD) de l'Organisation des Nations Unies, qui vise à terme à "Garantir l'accès de tous à des sources d'eau potable et à l'assainissement ». Son objectif est de caractériser le système aquifère sur le plan structurale et hydrodynamique, c'est-à-dire de préciser sa structure ses paramètres hydrodynamiques et sa piézométrie.

\section{Présentation du milieu d'étude}

La carte de localisation du milieu d'étude (BSK) est présentée sur la figure 1. Il s'agit du Modèle Numérique de Terrain (MNT) issue de la source CGIAR-CSI v4.1 90m x 90m (Gbewezoun, 2017). Avec une superficie d'environ $8700 \mathrm{Km}^{2}$ (Achidi et al., 2012), le BSK est situé au Nord-Est du Bénin (Figure 1) et représente la partie béninoise du vaste bassin sahélien de l'Iullemeden, qui est connu au Nigeria, à l'Est de la zone d'étude, sous le nom de bassin de Sokoto. Il est baigné pour l'essentiel par le bassin versant du fleuve Sota, affluent du fleuve Niger (Alidou, 1983). Il s'étend sous un climat de transition entre le climat du type tropical et celui du type sahélien. Sur la période 1985-2015, la pluviométrie moyenne annuelle à la station synoptique de Kandi (Figure 1) est de 1002 mm, la température maximale moyenne annuelle, de $34,7^{\circ} \mathrm{C}$ et l'Evapotranspiration Potentielle annuelle moyenne de 1703 mm (Gbewezoun, 2017). La végétation dans le bassin est généralement composée, en conséquence, de formations caractéristiques de la zone climatique soudanienne, mais aussi de certaines espèces plus spécifiquement sahéliennes, qui ne se rencontrent qu'au Nord du bassin (Alidou, 1983).

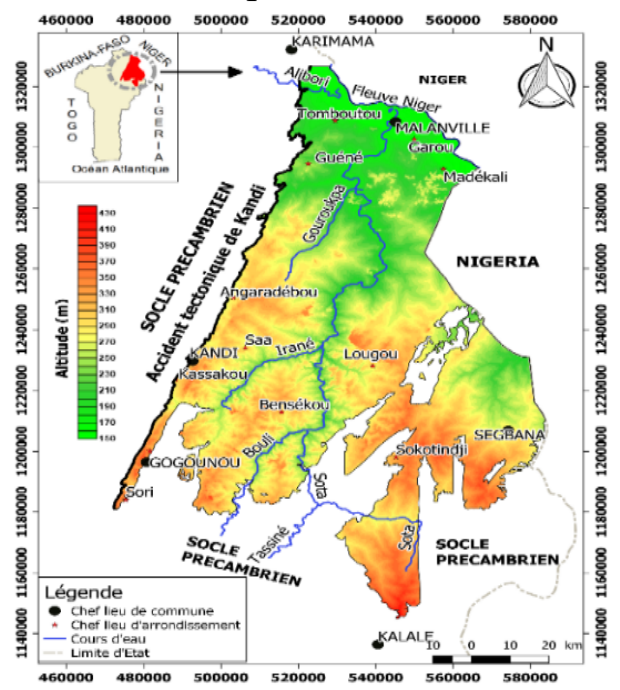

Figure 1. Carte de de localisation de la zone d'étude avec le réseau hydrographique et le MNT issu de la source CGIAR-CSI v4.1 90m x 90m

Sur le plan géomorphologique, les dépôts du BSK épousent remarquablement la topographie molle du socle dans la région, en formant une 
vaste plaine légèrement inclinée vers le Nord (Boukari, 2007). Le BSK se présente comme une plaine drainée et plus ou moins vallonnée par la Sota et ses affluents. A l'opposé, cet aplanissement général est, par endroits, interrompu par des buttes-témoins de dépôts dits du Continental Terminal, plus ou moins protégées par des cuirasses ferrugineuses (SERHAU, 1992).

Le BSK est caractérisé principalement par des sols ferrugineux tropicaux plus ou moins sableux. Les vallées du fleuve Niger et de son affluent la Sota, sont couvertes par des sols alluviaux hydromorphes (Azontonde, 1991).

\section{Contexte géologique et hydrogéologique}

La stratigraphie du BSK a été décrite successivement par Alidou (1983), Alidou et al. (1983), Alidou (1987), Seilacher et Alidou (1988), IRB (1989), Alidou et al. (1991) et Konaté (1996), à partir de données découlant des observations de terrain et de synthèses cartographiques. Il s'agit pour l'essentiel de formations cambro-siluriennes. Chronologiquement, elles commencent par une formation sédimentaire du Cambrien et/ou Ordovicien supérieur selon les auteurs (Figures 2, 3 et 4), connue sous le nom de Formation de Wèrè (FW) (Alidou, 1991, Figure 3), d'origine continentale. Elle débute par un faciès conglomératique d'une épaisseur maximale d'environ 150 m à l'affleurement (faciès K1 de IRB, 1989, Figure 4), et se termine par un faciès gréseux grossier, dont l'épaisseur maximale est d'environ 350 m (faciès K2 de IRB, 1989, Figure 4).

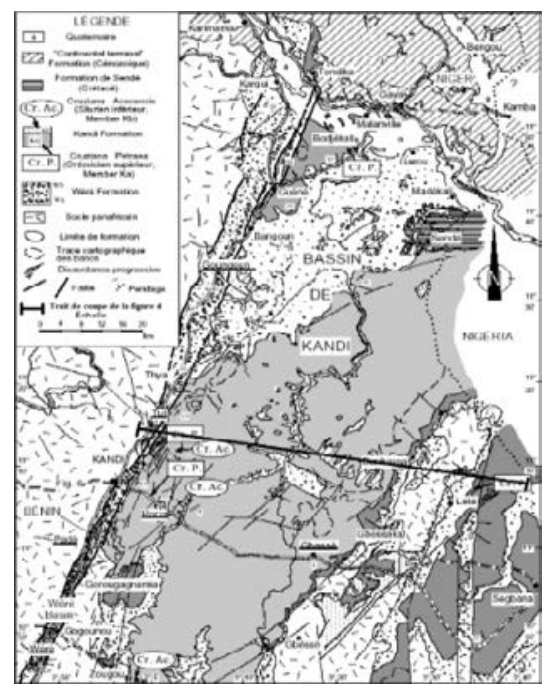

Figure 2. Carte géologique du bassin sédimentaire de Kandi (Konaté, 1996)

La FW est surmontée en discordance de ravinement par un ensemble de dépôts marins dénommé Formation de Kandi (FK) (Alidou et al., 1991), 
datée de l’Ordovicien Terminal - Silurien Inférieur (Konaté, 1996), qui affleure largement pour couvrir la plus grande partie du bassin (Figure 2). Avec une puissance de l'ordre de $75 \mathrm{~m}$, elle repose directement sur le socle précambrien dans certains secteurs sur sa bordure sud et est constituée d'un matériel gréseux fin et silteux, présentant parfois des intercalations de niveaux gréseux grossiers (Konaté, 1996) (Figure 3).

Une importante lacune stratigraphique sépare les derniers dépôts marins du Silurien inférieur de la FK, des dépôts sus-jacents continentaux du Crétacé inférieur, connus sous le nom de Formation de Sendé (FS) (Alidou et al., 1991) (Figure 3).

Les dépôts détritiques de la FS sont localisés seulement au Nord-Est du bassin (Figure 2) et reposent en discordance de ravinement sur ceux sousjacents (FW et FK). La FS, de faible extension et de faible puissance (20 à 30 $\mathrm{m})$ est également en continuité avec les sédiments du bassin de Sokoto au Nigéria (Konaté, 1996).

La FS est surmontée par une formation dite du "Continental terminal" (CT), correspondant à de "vastes épandages détritiques continentaux, remaniés" (faciès sidérolithique), d'âge post-Eocène moyen et antéQuaternaire (Lang et al., 1986 et 1990). Au Bénin, cette formation se développe surtout à l'Ouest, le long du fleuve Niger (Figure 3) (Konaté, 1996).

Enfin, la Formation du Quaternaire (FQ), également peu développée dans le bassin, termine la série sédimentaire. Elle tapisse la vallée du Niger et, dans une certaine mesure, celle de la Sota et coiffe certaines cuirasses latéritiques, avec une faible épaisseur, pouvant atteindre au plus $100 \mathrm{~m}$ (Alidou, 1983). Elle est caractérisée par des épandages détritiques et des accumulations chimiques, surtout ferrugineuses. Des concrétions calcaires y sont toutefois présentes. Ce qui témoignerait d'un épisode climatique proche du type méditerranéen (Alidou, 1983). Cet auteur considère que les grands traits de la morphologie actuelle du bassin ont été imprimés au Quaternaire.

Sur le plan tectonique, Konaté et al. (1994) concluent que la structuration du BSK est contrôlée par l'accident de Kandi, associé à un ensemble d'accidents secondaires d'orientation $\mathrm{N} 10^{\circ}-30^{\circ} \mathrm{E}$ à $\mathrm{N} 90^{\circ}-110^{\circ} \mathrm{E}$, et limitant des horsts de socle dans les secteurs de Poria, Gbéssé et Lété. Cet accident, principale structure cisaillante de la sous-région, est le prolongement méridional de l'accident $4^{\circ} 50$ du Hoggar (Sahara algérien) et possède un autre tronçon dans le Nord-Est du Brésil (Konaté, 1996). Selon Konaté et al. (2006), le contexte tectonique est relié à un demi-graben dissymétrique et le BSK peut être défini comme un bassin en demi-graben (Figure 4).

Enfin, le BSK n'étant pas encore entièrement traversé par des forages profonds, est mal connu sur le plan hydrogéologique, d'autant plus qu'il a été le siège d'importants épisodes tectoniques qui ont rendu sa structure complexe. L'entièreté de l'épaisseur du BSK, non encore mécaniquement 
reconnue pourrait, au maximum, atteindre, voire dépasser 600 m (IRB, 1989 et Konaté, 1996) (Figures 3 et 4). C’est pour contribuer à combler cette méconnaissance hydrogéologique du bassin que la présente étude de caractérisation structurale et hydrodynamique est menée. Elle ne concerne que la tranche superficielle exploitée du système aquifère du bassin dont il est admis qu'il présente dans son ensemble une épaisseur relativement importante. Dans ce qui suit, le matériel et la méthodologie sont décrits.

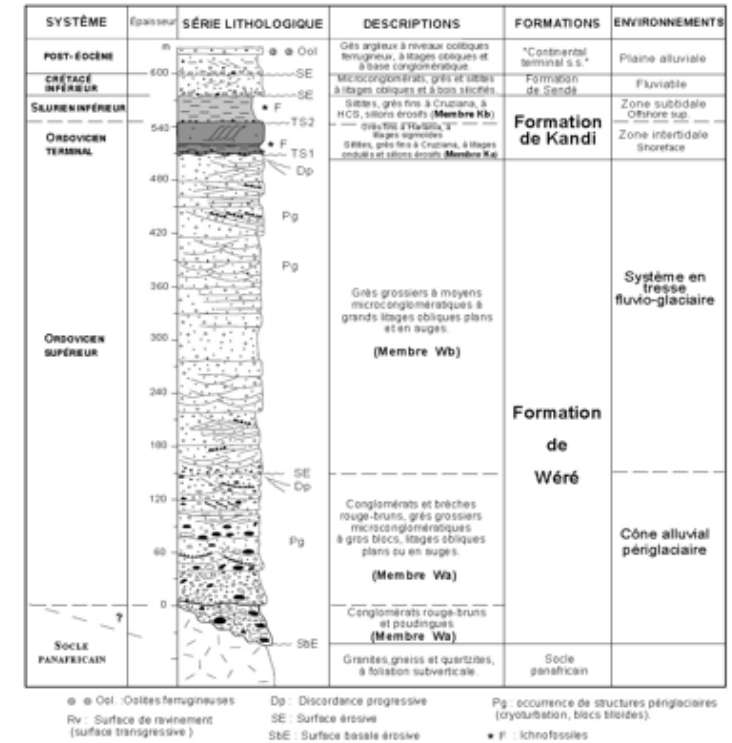

Figure 3. Colonne lithostratigraphique de la série paléozoïque de Kandi (Konaté et al., 2006)

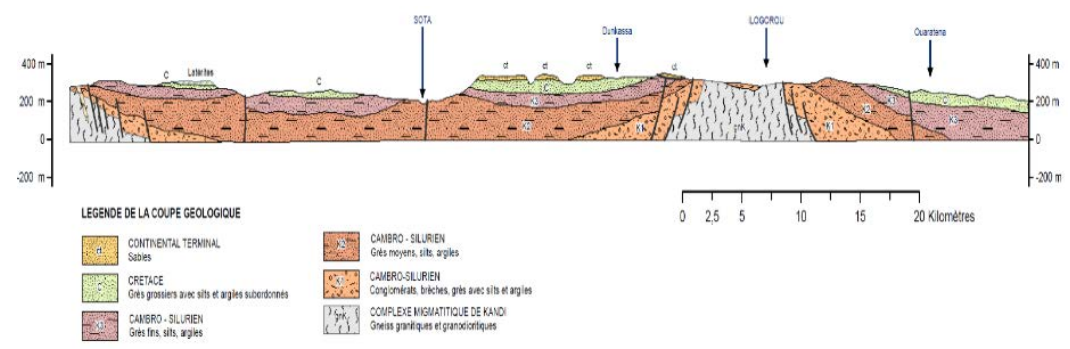

Figure 4. Coupe géologique du bassin sédimentaire de Kandi (IRB 1989 in Achidi et al., 2012)

\section{Outils, données et méthodes Outils et données}

Les outils utilisés sont constitués d'un GPS (Global Positioning System) de marque Garmin et Extrex pour la prise des coordonnées géographique sur le terrain, d'une sonde piézométrique (de marque SEBA Hydrometria) pour la mesure des niveaux statiques, de logiciels Excel et 
Scilab 5.5.2 pour le traitement des données, QGIS 2.8.3 et Surfer 11 pour la réalisation des cartes, GMS 8.0 pour la visualisation des logs stratigraphiques et GIMP 2.8.16 pour l'agencement des images scannées des corrélations lithostratigraphiques et des coupes hydrogéologiques réalisées sur du papier millimétré.

Les données collectées sur la zone d'étude se composent de cartes de terrain (cartes topographiques feuilles Kandi, Gaya, Dunkassa et Bembèrèkè ; géologiques feuilles Malanville et Kandi (1989) puis Dunkassa et Bembèrèkè (1995) (1/200000), modèle numérique de terrain téléchargé en 2016 à partir du site ftp://srtm.csi.cgiar.org), quelques altitudes des bornes géodésiques de l’Institut Géographique National (IGN) du Bénin, de logs lithologiques et côtes des crépines des forages sélectionnés pour les coupes hydrogéologiques, de données de pompage d'essai de courtes (2009) et longues durées (2013).

En tout, 176 logs stratigraphiques (de forages et piézomètres) ont été collectés, parmi lesquels ont été sélectionnés ceux ayant servi à la réalisation des coupes hydrogéologiques (Figure 5). Les données de pompages d'essai collectées ont servi à l'estimation des paramètres hydrodynamiques du système aquifère étudié (transmissivité et perméabilité). Les niveaux statiques mesurés dans 404 et 408 puits (à grand diamètre et simple par endroit en absence de ceux à grand diamètre) (Figure 5) respectivement en période des hautes eaux (août 2016) et en période des basses eaux (avril 2017). Ces niveaux statiques ont permis de réaliser des cartes piézométriques du système aquifère qui sont présentées et analysées plus loin.

\section{Méthodes}

\section{Réalisation des coupes hydrogéologiques}

Pour caractériser la structure et la géométrie du système aquifère capté par les forages d'exploitation nous avons, dans un premier temps, procédé à l'identification géologique de ce système, par la réalisation de trois (03) coupes, dont l'une (coupe $\mathrm{AB}$ ) longitudinale à la direction d'écoulement générale de la nappe (direction SSW-NNE) et les deux autres (coupes CD et $\mathrm{EF}$ ) transversales à cette direction (direction WNW-ESE) (Figure 5).

Pour réaliser ces coupes (Gbewezoun, 2017) les logs stratigraphiques des forages ont été projetés dans QGIS 2.8.3. Puis, les profils topographiques et les corrélations lithostratigraphiques entre les différents logs stratigraphiques des forages ont été réalisés sur du papier millimétré et à l'aide de cartes topographiques citées plus haut ; les crépines et les niveaux statiques (mesurés lors de la réalisation des forages) ont été ensuite reportés aux voisinages des forages et, enfin, ces niveaux ont été corrélés pour l'obtention de la ligne piézométrique de l'horizon capté ; les coupes obtenues sont scannées et agencées avec GIMP 2.8.16 et digitalisées dans QGIS 2.8.3 pour être exportées sous forme d'image. 


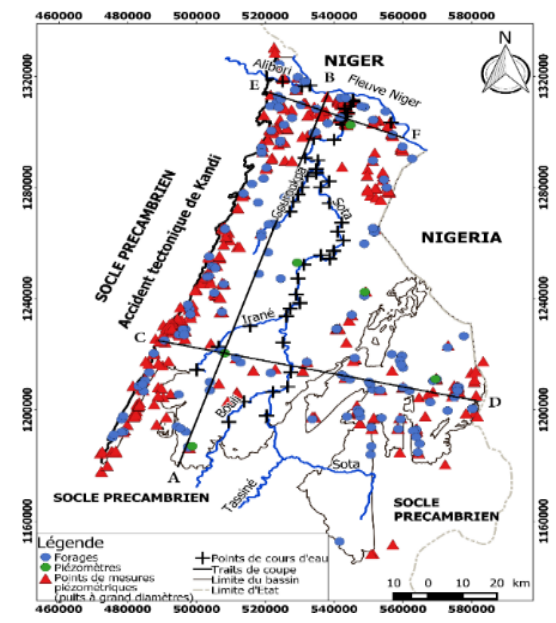

Figure 5. Localisation des traits de coupe et des ouvrages exploités pour l'étude

\section{Estimation des paramètres hydrodynamiques}

Quelques données issues des pompages d'essai de courtes durées comme de longues durées ont été utilisées pour évaluer, en divers points, les paramètres hydrodynamiques ( $\mathrm{T}$ et $\mathrm{K}$ ) du système aquifère étudié. Il s'agit d'avoir une idée des valeurs de ces paramètres, car trop peu de données exploitables sont disponibles (08 essais de courte durée exécutés dans 8 localités de trois communes du bassin et 2 essais de longue durée dans deux localités d'une même commune).

Les données de pompages d'essai de longue durée ont servi à calculer les transmissivités (T) par la méthode de remontée de Theis (1935), selon la formule :

$$
T=\frac{2,3 Q}{4 \pi \Delta s^{\prime}}
$$

Avec : T ( $\left.\mathrm{m}^{2} / \mathrm{s}\right)$ la transmissivité ; $\mathrm{Q}\left(\mathrm{m}^{3} / \mathrm{s}\right)$ le débit de pompage constant ; $\Delta \mathrm{s}^{\prime}(\mathrm{m})$ la variation de rabattement résiduelle $\mathrm{s}$;

Les données de courte durée (pompages par paliers de débits constants) ont été utilisées pour le calcul des transmissivités, mais par la méthode de descente de Jacob (Kruseman et al., 1974) selon la formule:

$$
T=\frac{2,30}{4 \pi d}=\frac{0,183}{d}
$$

Avec : $\mathrm{d}=\Delta(\mathrm{s} / \mathrm{Q})$, la pente de la droite d'ajustement obtenue pour $s / \mathrm{Q}=\mathrm{f}(\mathrm{t}) ; \mathrm{Q}\left(\mathrm{m}^{3} / \mathrm{s}\right)$ le débit de pompage constant, $\mathrm{s}(\mathrm{m})$ le rabattement, $\mathrm{t}(\mathrm{min})$ le temps

Le coefficient de perméabilité (K) est déduit de la transmissivité par la formule utilisée par Alassane (2004):

$$
K=\frac{\mathrm{T}}{\mathrm{LC}}
$$


Avec : K (m/s) le coefficient de perméabilité ; T (m²/s) la transmissivité ; LC (m) la longueur de la crépine, c'est-à-dire l'épaisseur d'aquifère captée.

Les coefficients d'emmagasinement n’ont pas été calculés, car les données ne sont disponibles qu’au niveau des ouvrages en pompage.

\section{Cartographie de la profondeur et de la piézométrie de la nappe Cartographie de la profondeur de la nappe}

Le processus de réalisation des cartes de niveaux statiques peut être décliné comme suit. Les niveaux statiques (NS) ont été mesurés dans 404 et 408 puits (à grand diamètre) respectivement en période des hautes eaux (août 2016) et en période des basses eaux (avril 2017), en prenant d'abord la profondeur de l'eau par rapport à la margelle du puits avec une sonde piézométrique, puis en soustrayant la hauteur de cette margelle. Les coordonnées géographiques ( $\mathrm{X}$ et $\mathrm{Y}$ ) de ces puits ont été également relevées avec un GPS.

Pour le tracé des courbes d'égal niveau statiques, la méthode d'interpolation choisie est le krigeage, car les résultats peuvent être contrôlés avec les variogrammes des distances. D’après Matheron (1965), le variogramme théorique n'est représentatif du variogramme empirique qu'au voisinage de l'origine. Le modèle sphérique est celui qui s'ajuste le mieux à nos valeurs de niveaux statiques pour les deux saisons de mesures (Figure 6). Au niveau des variogrammes (Figure 6), la distance maximale de corrélation entre les points (portée) pour les basses eaux (15 $500 \mathrm{~m}$ ) est supérieure à celle de corrélation pour les hautes eaux (10 $000 \mathrm{~m})$. Cette différence s'expliquerait par le fait que les niveaux d'eau ne sont pas perturbés par les précipitations pendant les basses eaux.
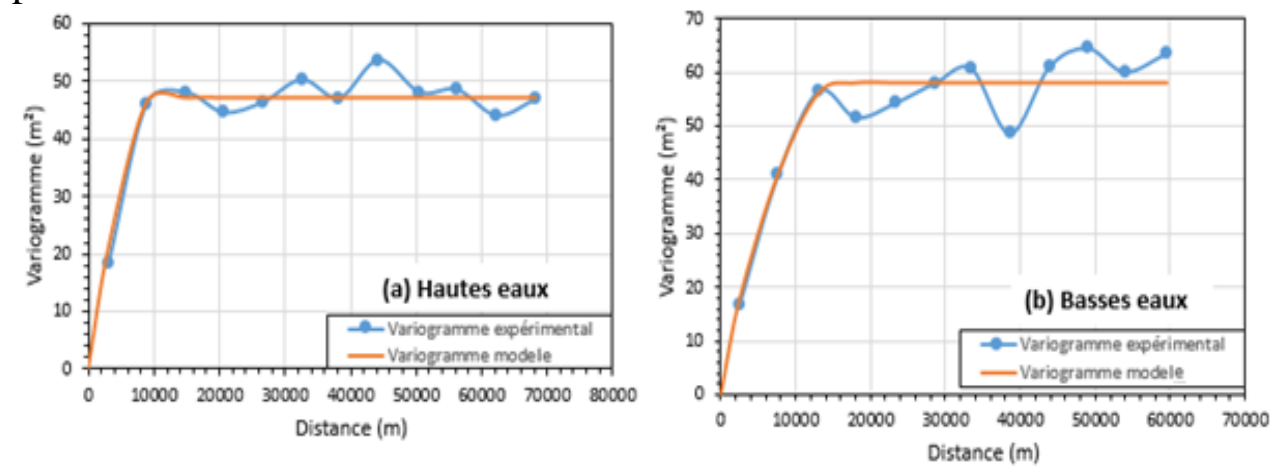

Figure 6. Variogrammes des distances ajustés pour les niveaux statiques avec a: Hautes eaux et b: Basses eaux. Il est à noter que les variogrammes ne présentent pas de saut abrupt à l'origine (effet de pépite) perceptible, cela indique une bonne correlation entre couples de valeurs prises en deux sites très proches. Autrement dit, il y a une grande ressemblance entre les valeurs de niveaux statiques régionalisées très voisines. 
Les formules du modèle sphérique d'ajustement pour les deux périodes (hautes et basses eaux) sont les suivantes:

- pour les hautes eaux
$\left\{\begin{array}{c}\gamma_{H E}(h)=47 *\left[1,5 *\left(\frac{h}{10000}\right)-0,5 *\left(\frac{h^{2}}{10000^{2}}\right)\right] \text { si } 0<h \leq 10000 \\ \gamma_{H E}(h)=47 \text { si } h>10000\end{array}\right.$

- $\quad$ pour les basses eaux

$$
\left\{\begin{array}{c}
\gamma_{B E}(h)=5+53 *\left[1,5 *\left(\frac{h}{15500}\right)-0,5 *\left(\frac{h^{2}}{15500^{2}}\right)\right] \text { si } 0<h \leq 15500 \\
\gamma_{B E}(h)=58 \text { si } h>15500
\end{array}\right.
$$

avec $h$ la distance entre deux points, $\gamma_{H E}(h)$ le variogramme modèle des hautes eaux et $\gamma_{B E}(h)$ celui des basses eaux, 10000 , la portée (en m) du variogramme des hautes eaux et 15500 la portée (en m) du variogramme des basses eaux.

Etant donné que l'ajustement du variogramme est priorisé sur la première partie du graphique (portée du variogramme), les critères d'appréciation de l'ajustement peuvent être calculés en considérant les valeurs du variogramme des distances situées dans cette première partie. Le premier critère utilisé pour évaluer la pertinence de l'ajustement du variogramme est le coefficient de Nash NSE (Nash-Sutcliffe Efficiency) (Nash et Sutcliffe, 1970) utilisé par Lawin (2007) et le RSR (RMSE observations Standard deviation Ratio) (Moriasi et al., 2007) utilisé par Gbewezoun (2017), tels que ;

$$
\begin{gathered}
N S E=1-\left[\frac{\sum_{\mathrm{i}=1}^{\mathrm{n}}\left(Y_{i}{ }^{\text {obs }}-\mathrm{Y}_{i}{ }^{\mathrm{cal}}\right)^{2}}{\sum_{\mathrm{i}=1}^{\mathrm{n}}\left(Y_{i}{ }^{\text {obs }}-\overline{\mathrm{Y}}^{\mathrm{obs}}\right)^{2}}\right] \\
R S R=\frac{R M S E}{S T D E V_{\text {obs }}}=\frac{\sqrt{\sum_{\mathrm{i}=1}^{\mathrm{n}}\left(Y_{i}{ }^{\text {obs }}-\mathrm{Y}_{i}{ }^{\mathrm{cal}}\right)^{2}}}{\sqrt{\sum_{\mathrm{i}=1}^{\mathrm{n}}\left(Y_{\mathrm{i}}{ }^{\mathrm{obs}}-\overline{\mathrm{Y}}^{\mathrm{obs}}\right)^{2}}} \text { avec } R M S E=\sqrt{\frac{1}{n} \sum_{i=1}^{\mathrm{n}}\left(Y_{i}^{\text {obs }}-Y_{i}{ }^{\mathrm{cal}}\right)^{2}}
\end{gathered}
$$

$Y_{i}^{\text {obs }}$ est la ième observation pour le constituant évalué, $\mathrm{Y}_{i}^{\text {cal }}$ est la ième valeur estimée pour

le constituant évalué, $\overline{\mathrm{Y}}^{\mathrm{obs}}$ est la moyenne des données d'observation pour le constituant

évalué et $n$ le nombre total d'observation. Il faut noter que dans le cas de cette étude le constituant évalué est soit le variogramme, l'altitude, le niveau statique de l'eau ou le niveau piézométrique de la nappe.

L'intérêt du RSR est qu'il normalise le RMSE (Root Mean Square Error - erreur quadratique moyenne) par l'écart type des constituants concernés (Moriasi et al., 2007). Les valeurs des critères de comparaison des variogrammes expérimentaux avec ceux modélisés sont présentées dans le tableau 1. 
Tableau 1. Paramètres de comparaison des valeurs des variogrammes brutes avec celles des modèles pour les niveaux statiques

\begin{tabular}{|c|c|c|}
\hline Période & NSE & RSR \\
\hline Hautes eaux (août 2016) & 0,90 & 0,29 \\
\hline Basses eaux (avril 2017) & 0,87 & 0,33 \\
\hline
\end{tabular}

Pour comparer les valeurs des niveaux statiques mesurées avec celles estimées par krigeage, nous avons également utilisé le coefficient de Nash et le RSR comme présenté dans le tableau 2. Ces valeurs remplissant aussi les conditions de Moriasi et al. (2007) $(0,75<\mathrm{NSE} \leq 1$ et $0 \leq \mathrm{RSR} \leq 0,50)$, indiquent que les niveaux statiques sont très bien estimées aux points de mesure par la méthode d'interpolation choisie.

Tableau 2. Valeurs des paramètres de comparaison des valeurs de niveaux statiques mesurées avec celles estimées par krigeage

\begin{tabular}{|c|c|c|}
\hline Période & NSE & RSR \\
\hline Hautes eaux (août 2016) & 0,92 & 0,28 \\
\hline Basses eaux (avril 2017) & 0,90 & 0,31 \\
\hline
\end{tabular}

Les cartes d'écarts types d'estimation ainsi que les droites de corrélation entre les niveaux statiques mesurés et ceux estimés sont présentés sous forme de cartouches sur les cartes des figures 10 et 11 , avec leur coefficient de détermination $\mathrm{R}^{2}$ respectif.

\section{Cartographie de la piézométrie de la nappe}

L'établissement des cartes piézométriques a été entrepris en trois étapes: la détermination des altitudes des points de mesure, le calcul des niveaux piézométriques et l'interpolation des niveaux piézométriques calculés.

\section{Détermination des altitudes des points de mesure}

Le niveau piézométrique est le niveau statique rapporté au niveau 0 moyen de la mer. Dans l'impossibilité de niveler tous nos points de mesure et étant donnée la faible précision des altitudes $Z$ relevées par le GPS sur le terrain, il a été procédé à leur détermination en utilisant l'équation de Gbewezoun (2017). Cette équation a été établit à travers une corrélation effectuée entre des valeurs d'altitude des bornes géodésiques de l’IGN Bénin (Figure 7) se trouvant à l'intérieur et à proximité de la zone d'étude, et des valeurs d'altitudes de ces bornes géodésiques extraites d'un Modèle Numérique de Terrain (MNT) CGIAR-CSI v4.1 (MNT de résolution spatiale $90 \mathrm{~m} \times 90 \mathrm{~m}$, téléchargé sur le site ftp://srtm.csi.cgiar.org déjà rapporté plus haut). De plus, les résultats des travaux de Rexer et Hirt (2014), stipulent que le MNT issu de CGIAR-CSI à une meilleure précision comparativement aux MNT d'autres sources appliquées à des points de référence en Australie. 


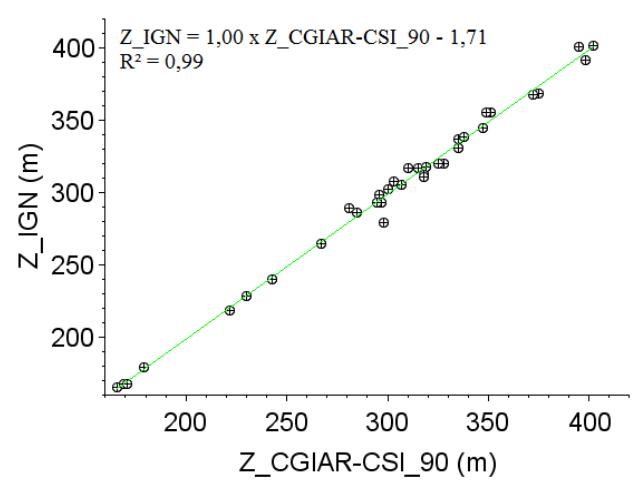

Figure 7. Droite de corrélation entre les altitudes de l'IGN et

celles de la source CGIAR-CSI v4.1 90m x 90m , ftp://srtm.csi.cgiar.org (Gbewezoun, 2017)

Gbewezoun (2017) a utilisé l'équation qu'il a établi pour réaliser le MNT de la zone d'étude présenté sur la figure 1 . La figure 7 présente la droite de corrélation entre les altitudes $\mathrm{Z}$ (en $\mathrm{m}$ ) de l'IGN et les altitudes $Z_{\text {CGIAR-CSI-90 }}$ (en $\mathrm{m}$ ) du MNT choisi. L'équation de cette droite est (Gbewezoun, 2017):

$$
Z=1,00 \times Z_{C G I A R-C S I-90}-1,71
$$

C’est cette équation qui a été utilisée pour estimer les altitudes de nos points de mesure dans notre zone d'étude.

En outre, seuls quelques points de cours d'eau ont pu être mesurés sur le terrain, essentiellement dans la portion aval de la Sota, l'accès à ce cours d'eau et ses affluents majeurs vers l'amont n'ayant pas été possible (zone de forêt ou inhabitée). Or, les coupes hydrogéologiques de la figure 9 montrent que les vallées de ces cours d'eau (Sota et ses affluents majeurs) interceptent la surface de la nappe phréatique étudiée, tendant à confirmer l'existence d'une interconnexion hydraulique entre la nappe et ces plans d'eau de surface, comme invoqué déjà par Kpegli et al. (2015). En conséquence, pour améliorer la précision des cartes à établir, les points d'eau de surface reconnus sur le terrain ont été complétés par quelques points récupérés le long de ces cours d'eau, à partir de la source d'altitude utilisée pour estimer les altitudes des points de mesures des niveaux statiques (Figure 5).

\section{Calcul des niveaux piézométriques}

Pour le calcul des niveaux piézométriques $N P$ (en m) des points de mesure de niveaux statiques, nous avons pris en compte les altitudes $Z$ (en m) de ces points déterminées à partir de la formule suivante:

$$
N P=Z-N S_{/ s o l}
$$


En intégrant (8) dans (9) et en remplaçant Z par sa valeur, (9) devient :

$$
N P=\left(1,00 \times Z_{C G I A R-C S I-90}-1,71\right)-N S_{/ s o l}
$$

Avec $N P(\mathrm{~m})$ le niveau piézométrique ; $Z$ (m) l'altitude estimée du point de mesure; $Z_{\text {CGIAR-CSI-90 }}(\mathrm{m})$ est la valeur de l'altitude du point de mesure provenant du MNT de la source CGIAR-CSI v4.1 $90 \mathrm{~m} \times 90 \mathrm{~m}$ et $N S_{/ s o l}(\mathrm{~m})$ le niveau statique mesuré par rapport au sol.

\section{Interpolation des niveaux piézométriques}

La procédure d'interpolation des niveaux piézométriques est la même que celle utilisée plus haut pour les niveaux statiques. Trois différentes interpolations ont été réalisées qui ont permis d'établir trois cartes piézométriques, à savoir celle des hautes eaux (août 2016), celle des basses eaux (avril 2017) et celle correspondant aux niveaux piézométriques moyens (entre les hautes eaux de 2016 et les basses eaux de 2017). Les variogrammes des trois interpolations sont présentées sur la figure 8.

Le modèle Puissance est celui qui s'ajuste le mieux à nos valeurs de niveaux piézométriques. Les formules d'interpolation correspondantes aux trois cas de figure dans le cas présent sont les suivantes :

- pour les hautes eaux

$\gamma_{H E}(h)=110+5,15 \times 10^{-04} \times h^{1,4}$

- pour les basses eaux

$$
\gamma_{B E}(h)=30+4,24 \times 10^{-03} \times h^{1,2}
$$

- pour les moyennes eaux

$\gamma_{M E}(h)=2,39 \times 10^{-03} \times h^{1,27}$

Avec $h$ la distance entre deux points, $\gamma_{H E}(h)$ le variogramme modèle pour les hautes eaux, $\gamma_{B E}(h)$ celui pour les basses eaux et $\gamma_{M E}(h)$ celui pour les moyennes eaux.
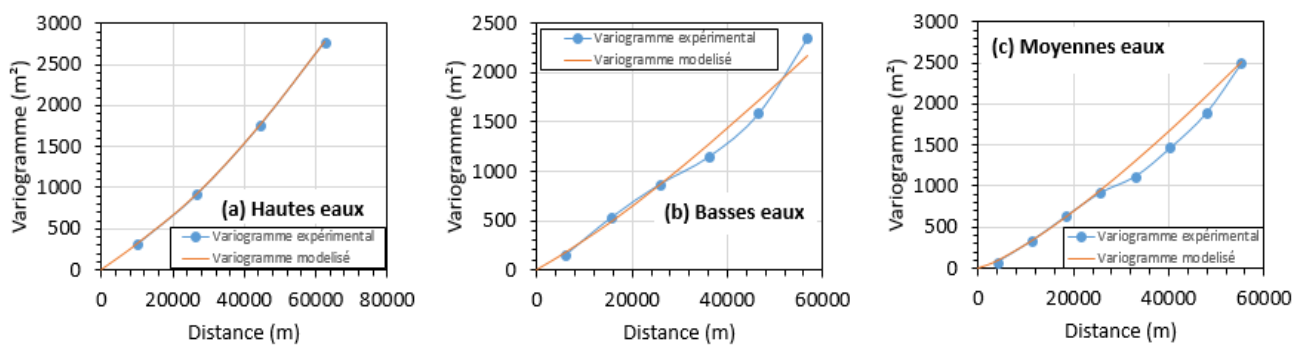

Figure 8. Variogrammes des distances ajustés pour les niveaux piézométriques de hautes eaux (a), basses eaux (b) et moyennes eaux (c)

Le tableau 3 présente les valeurs des paramètres de comparaison calculés pour les variogrammes relatifs aux niveaux piézométriques. 
Tableau 3. Valeurs des paramètres de comparaison des variogrammes bruts et des modèles pour les niveaux piézométriques

\begin{tabular}{|c|c|c|}
\hline Période & NSE & RSR \\
\hline Hautes eaux (août 2016) & 0,99 & 0,06 \\
\hline Basses eaux (avril 2017) & 0,98 & 0,13 \\
\hline Moyennes eaux & 0,97 & 0,15 \\
\hline
\end{tabular}

Les paramètres de comparaison des valeurs de niveaux piézométriques mesurés avec celles estimées par krigeage sont reportés dans le tableau 4.

Tableau 4. Valeurs des paramètres de comparaison des valeurs de niveaux piézométriques mesurées et celles estimés par krigeage

\begin{tabular}{|c|c|c|}
\hline Période & NSE & RSR \\
\hline Hautes eaux (août 2016) & 0,99 & 0,10 \\
\hline Basses eaux (avril 2017) & 0,99 & 0,06 \\
\hline Moyennes eaux & 0,99 & 0,03 \\
\hline
\end{tabular}

Les cartes d'écarts types d'estimation, ainsi que les droites de corrélation entre les niveaux piézométriques mesurés et ceux estimés, sont présentés sous forme de cartouches sur les cartes des figures 13, 14 et 15, avec leur coefficient de détermination $\mathrm{R}^{2}$ respectif.

\section{Résultats}

\section{Identification lithostratigraphique de l'aquifère superficiel}

A l'échelle du BSK, pour identifier l'aquifère phréatique, trois (03) corrélations lithostratigraphiques ont été effectuées sur, environ, les 80 premiers mètres d’épaisseur du bassin traversés par les ouvrages existants, dont deux suivant la direction WNW-ESE (coupe CD au Sud et coupe EF au Nord) et la troisième suivant la direction SSW-NNE (coupe AB). Les traits de coupe suivant lesquelles ces corrélations ont été faites sont indiqués sur la figure 5. Les coupes hydrogéologiques obtenues sont présentées sur la figure 9. Elles mettent en évidence les différentes couches traversées par les forages. La couche la plus ancienne est composée de conglomérats et grès. Elle correspondrait à la Formation de Wèrè décrite plus haut. Elle affleure au Sud du bassin à la faveur d'un horst et repose directement sur le socle précambrien. Cette première couche est surmontée dans la plus grande partie du bassin par une couche de grès plus ou moins argileux correspondant vraisemblablement au premier sous-ensemble de la Formation de Kandi. Elle est à son tour surmontée par endroits par une couche d'argilite gréseuse épaisse de $40 \mathrm{~m}$ maximum, qui correspondrait au second sous-ensemble de la Formation de Kandi. Vers le Nord, dans la vallée du Niger et le cours aval de la Sota, affleure une dernière couche correspondant aux alluvions des deux fleuves (Figure 9a). Elle semble reposer directement sur la Formation de 
Kandi. Alors que l'épaisseur de ces différentes couches identifiées augmente du Sud du bassin vers le Nord, les couches correspondant à la Formation de Sendé et au CT sont d'extension négligeable et apparaissent très peu ou pas sur les coupes.

Du point de vue hydrogéologique, il apparaît clairement que c'est la couche correspondant à la Formation de Kandi qui est la plus largement traversée par les forages d'exploitation. Elle constitue l'essentiel de l'aquifère phréatique exploité (Figure 9). Mais vers l'Ouest et le Sud du bassin, où la Formation de Wéré est affleurante (Figures 9b et 9c), c'est cette dernière qui est reconnue par les forages et qui constitue l'aquifère phréatique. En raison de la faiblesse de son épaisseur ( $25 \mathrm{~m}$ environ en moyenne), la nappe phréatique de la couche des alluvions ne semble pas être captée par les forages (Figure 9a). En effet, ils la traversent complètement pour aller capter la nappe de la Formation de Kandi sous-jacente. Dans tous les cas, on observe une interconnexion hydraulique latérale et verticale entre les différents aquifères du système étudié. Donc, tout se passerait dans l'ensemble, sur le plan hydrodynamique, comme si on avait à faire à un aquifère unique, à nappe libre.

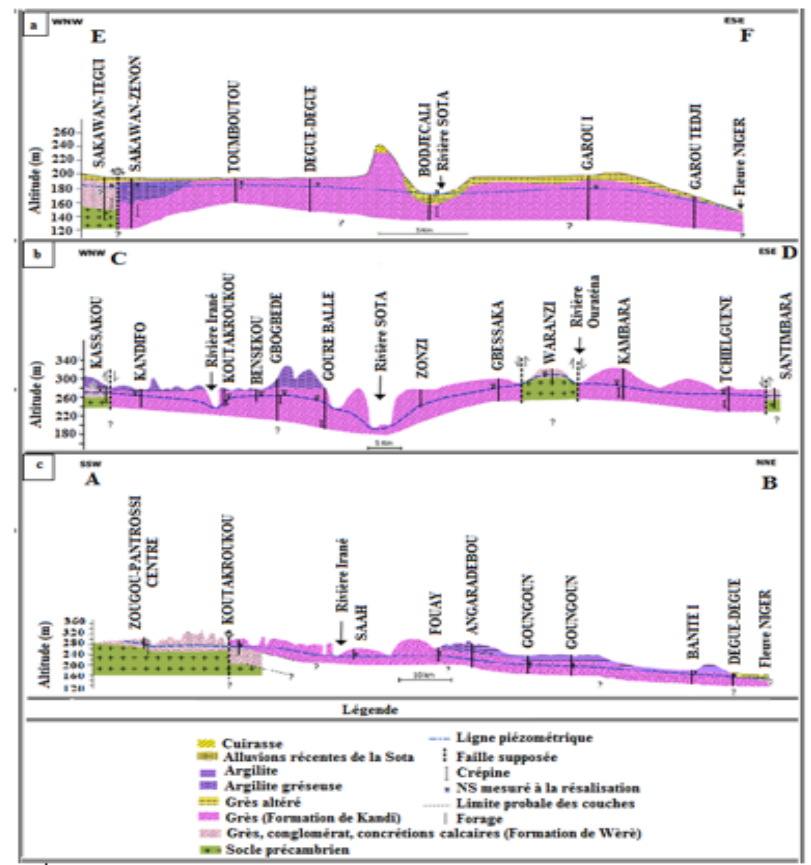

Figure 9. Coupes hydrogéologiques du bassin sédimentaire de Kandi suivant la direction WNW-ESE (coupes CD et EF de la Figure 5) et la direction SSW-NNE (coupe $\mathrm{AB}$ de la figure 5) 


\section{Paramètres hydrodynamiques de l'aquifère superficiel}

Les paramètres hydrodynamiques calculés pour l'aquifère superficiel identifié ci-dessus sont la transmissivité et le coefficient de perméabilité ou conductivité hydraulique, à partir des données issues des pompages de courte durée et de longue durée. Pour les pompages d'essai de courte durée, les résultats obtenus sont reportés dans le tableau 5.

Tableau 5. Paramètres calculés à partir des données de courte durée

\begin{tabular}{|c|c|c|c|c|}
\hline Ville & Localité & $\mathrm{T}\left(\mathrm{m}^{2} / \mathrm{s}\right)$ & Longueur Crépine LC $(\mathrm{m})$ & $\mathrm{K}(\mathrm{m} / \mathrm{s})$ \\
\hline Gogounou & Borodarou & $2,99 \mathrm{E}-05$ & 8,87 & $3,37 \mathrm{E}-06$ \\
\hline Ségbana & Guenelaga & $1,96 \mathrm{E}-04$ & 11,9 & $1,64 \mathrm{E}-05$ \\
\hline Ségbana & Owode II & $2,99 \mathrm{E}-04$ & 8,88 & $3,37 \mathrm{E}-05$ \\
\hline Ségbana & Goure-Kie & $3,51 \mathrm{E}-04$ & 11,87 & $2,95 \mathrm{E}-05$ \\
\hline Kandi & Angaradebou Gando & $3,91 \mathrm{E}-04$ & 11,9 & $3,29 \mathrm{E}-05$ \\
\hline Ségbana & Labari II & $4,71 \mathrm{E}-04$ & 8,9 & $5,29 \mathrm{E}-05$ \\
\hline Kandi & Zanloa & $7,70 \mathrm{E}-04$ & 26,55 & $2,90 \mathrm{E}-05$ \\
\hline Ségbana & Goure-Kani & $1,69 \mathrm{E}-03$ & 5,95 & $2,85 \mathrm{E}-04$ \\
\hline
\end{tabular}

Les valeurs de transmissivité sont de l'ordre de $10^{-4}$ dans l'ensemble sauf pour les essais de la localité de Borodarou $\left(10^{-5}\right)$ et de celle de GoureKani $\left(10^{-3}\right)$. Celles du coefficient de perméabilité sont de l'ordre de $10^{-5}$ dans l'ensemble, à l'exception des essais des mêmes localités de Borodarou $\left(10^{-6}\right)$ et de Goure-Kani $\left(10^{-4}\right)$. Les valeurs de transmissivité obtenues sont relativement faibles, ce qui n'est pas surprenant quand on sait que de faibles épaisseurs d'aquifère sont crépinées pour l'AEP d'hydraulique villageoise.

En ce qui concerne les pompages de longues durées, les résultats obtenus pour les deux paramètres sont consignés dans le tableau 6. Les valeurs de transmissivité sont de l'ordre de $10^{-2}$ pour les deux forages concernés. Celles du coefficient de perméabilité sont respectivement de $10^{-4}$ et de $10^{-3}$. Les valeurs de transmissivité sont de loin plus élevées car, contrairement au cas précédent, il s'agit ici de forages d'AEP d'hydraulique urbaine, qui sont crépinés sur une importante épaisseur d'aquifère. Les valeurs de conductivité hydraulique sont par contre relativement proches des précédentes, ce qui n'est pas surprenant, car elles ne sont pas dépendantes de l'épaisseur captée.

Au total, les valeurs de paramètres hydrauliques calculés avec les données d'essais de longues durées sont plus proches de la réalité, même si elles demandent à être densifiées et bien réparties pour être véritablement représentatives du système aquifère étudié.

Tableau 6. Transmissivité calculée à partir des données de longue durée

\begin{tabular}{|c|c|c|c|c|c|}
\hline Ville & Forage & $\mathrm{Q}\left(\mathrm{m}^{3} / \mathrm{h}\right)$ & $\mathrm{T}\left(\mathrm{m}^{2} / \mathrm{s}\right)$ & Longueur Crépinée LC $(\mathrm{m})$ & $\mathrm{K}(\mathrm{m} / \mathrm{s})$ \\
\hline Malanville & F1_A_SE1 & 23 & $3,08 \mathrm{E}-02$ & 23,76 & $1,30 \mathrm{E}-03$ \\
\hline Malanville & F3_B_SE5 & 23 & $1,17 \mathrm{E}-02$ & 28,31 & $4,13 \mathrm{E}-04$ \\
\hline
\end{tabular}




\section{Profondeur de la nappe phréatique et piézométrie Cartes de profondeur de la nappe phréatique}

Les cartes en courbes d'égale profondeur de la surface de la nappe obtenues pour les deux saisons sont présentées sur les figures 10 et 11 . Il ressort de ces cartes que les NS varient de 0 à environ $40 \mathrm{~m}$, les deux saisons confondues. En période des hautes eaux (Figure 10), les niveaux statiques les plus faibles, inférieurs à $10 \mathrm{~m}$ notamment, se rencontrent fréquemment dans la moitié sud du bassin et, de façon systématique, sur ses bordures nord (dans les environs du fleuve Niger) et ouest. Les NS moyens, compris entre 10 et $20 \mathrm{~m}$, se rencontrent dans la zone centrale du bassin. Les NS les plus élevés, supérieurs à $20 \mathrm{~m}$, se trouvent essentiellement vers l'extrême nord-est du bassin, sur une petite bande de terrain orientée NNW-SSE, s'étendant du Sud de la localité de Tomboutou à celle de Gogué Koara 2.

En période des basses eaux (Figure 11), la même distribution spatiale des NS s'observe, mais à la différence que les zones de valeurs faibles, inférieures à $10 \mathrm{~m}$, sont drastiquement réduites. Les NS, qui ont sensiblement augmenté, sont supérieurs à $10 \mathrm{~m}$ dans la quasi-totalité de la superficie du bassin. En outre, on note l'apparition d'un nouveau secteur de fortes valeurs de NS à l'Est de la localité d'Angaradébou, au niveau de certains points qui n'avaient pas été mesurés au cours de la campagne des hautes eaux d'août 2016.

\section{Hauteurs de battement de la nappe phréatique}

Les niveaux statiques (NS) ont été mesurés dans 404 et 408 puits respectivement en période des hautes eaux (août 2016) et en période des basses eaux (avril 2017). Mais, en tout 318 puits ont été concernés par les mesures durant les deux périodes. La figure 12 présente la carte de distribution spatiale de variation de niveau de la nappe superficiel dans ces puits entre les mois d'août 2016 et avril 2017. Il en ressort que les NS ont connu une diminution dans 291 puits (soit 91,51\%), une augmentation dans 24 puits (soit 7,55\%) et sont restés constants dans 3 puits (soit 0,94\%) localisés dans les villes de Malanville (au Nord) et de Ségbana (au Sud-Est).

La diminution de niveau est majoritairement comprise entre 0 et $5 \mathrm{~m}$, mais peut, dans certains puits, atteindre et dépasser $10 \mathrm{~m}$ voire, exceptionnellement, 20 et $30 \mathrm{~m}$. Les diminutions de niveau supérieures à $5 \mathrm{~m}$ concernent les puits situés dans les zones bordières méridionales et occidentales du bassin, aux abords du socle cristallin et cristallophyllien, c'està-dire, comme on le verra plus loin dans la suite, au niveau des zones de recharge de la nappe. Les stagnations et les remontées de niveaux se sont produites dans un nombre limités de puits situés, essentiellement, dans les zones de décharge de la nappe, c'est-à-dire les secteurs extrêmes nord et sudest du bassin. 


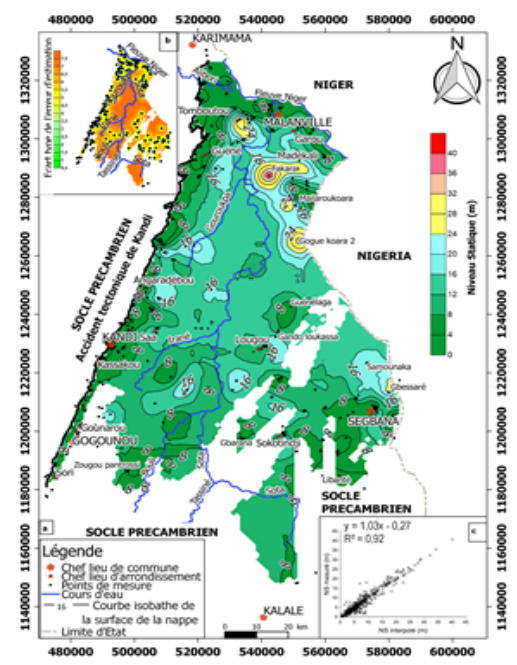

Figure 11. Carte bathymétrique de la surface de la nappe phréatique du bassin sédimentaire de Kandi (août 2016, période des hautes eaux)

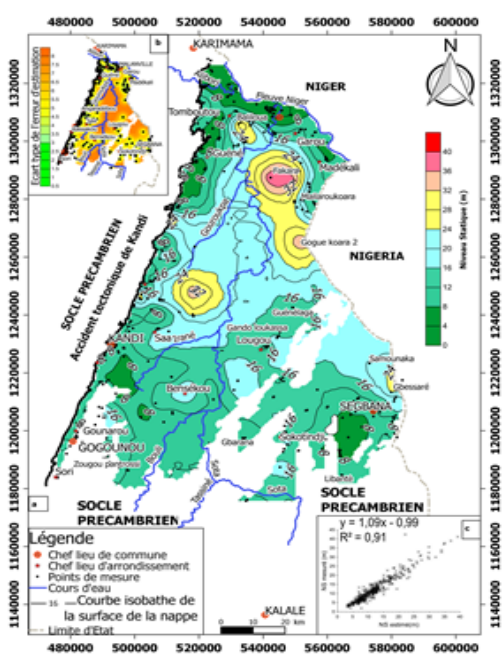

Figure 10. Carte bathymétrique de la surface de la nappe phréatique du bassin sédimentaire de Kandi (avril, 2017, période des basses eaux)

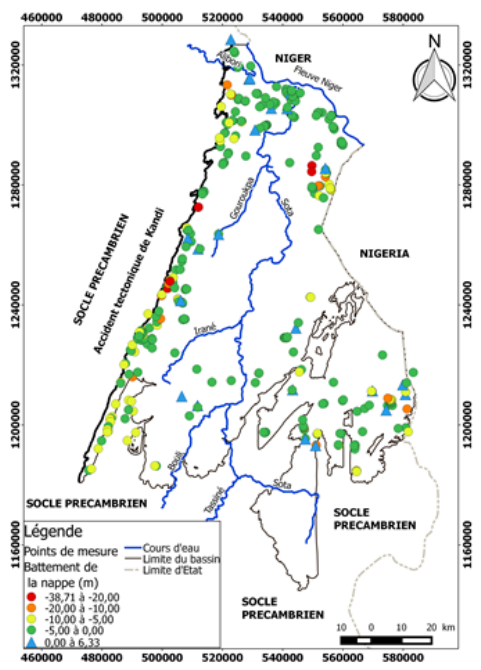

Figure 12. Carte de battement de la nappe phréatique du bassin sédimentaire de Kandi (2016-2017)

\section{Cartes piézométriques}

Bien que, pour l'essentiel, deux réservoirs d'eau souterraine (FW et FK) ont été identifiés dans le BSK, il est montré plus haut que ces deux réservoirs, en contact direct l'un et l'autre (Figure 4), formeraient un tout sur le plan hydrogéologique. Il existerait, par conséquent, une interconnexion hydraulique latérale et verticale entre les deux aquifères en présence et leurs surfaces piézométriques s'équilibrent pour former une seule et même surface (Figure 9). Tout se passe donc sur le plan hydrodynamique comme si l'on avait 
à faire à un aquifère unique, à nappe libre. C'est cette nappe qui a fait l'objet de la présente cartographie piézométrique.

Les cartes piézométriques dressées sont présentées sur les figures 13, 14 et 15. Elles montrent toutes que l'écoulement de la nappe phréatique étudiée tend à s'opérer de façon générale du Sud vers le Nord pour se décharger dans la vallée du Niger, et ceci, conformément au pendage régional des couches (Figures 4, 9b et 9c). Mais, les dépressions liées au réseau hydrographique de la Sota et ses affluents et à la structuration tectonique du bassin en demi-graben à géométrie synclinale, structuration ayant contrôlé étroitement les directions des apports sédimentaires (Konaté et al., 2006), font que cet écoulement échappe au contrôle strict par le pendage régional NNE (Figure 9c) des couches. Ainsi, on distingue des zones de recharge de la nappe localisées sur les bordures sud et ouest du bassin et des zones de drainage à forts gradients (4,3\%) d'une part, constituées par les vallées de la Sota et ses affluents à l'intérieur du bassin et, d'autre part, une zone de drainage à faibles gradients (2,8 \%o), constituée par le secteur de la vallée du Niger et du cours aval de la Sota, c'est-à-dire le secteur extrême-nord du bassin. On note toutefois la présence d'un dôme piézométrique centré sur la localité de Mailaroukoara vers l'extrême nord-est, et une dépression piézométrique centrée sur la localité de Samounaka au Sud-Est, les deux débordant sur le territoire nigerian. Les NP varient d'environ $310 \mathrm{~m}$ au Sud à $150 \mathrm{~m}$ au Nord pour les hautes eaux et d'environ $300 \mathrm{~m}$ au Sud à $150 \mathrm{~m}$ au Nord pour les basses eaux. Comme déjà signalé plus haut, le battement de la nappe est donc remarquable au Sud où il peut atteindre une dizaine de mètres, mais n'est que de quelques mètres au Nord dans la zone de décharge.

Par ailleurs, les cartes produites révèlent, selon la classification de Castany (1982) utilisée par Gbewezoun (2017), trois types principaux d'écoulement souterrain dans le BSK: (i) le type à module d'espacement décroissant (resserrement des isopièzes), traduisant un profil piézométrique parabolique, si bien que l'écoulement est non uniforme et le débit unitaire décroissant: c'est le type observé dans les zones de recharge, à l'Ouest et au Sud du bassin; (ii) le type à module d'espacement croissant, traduisant un profil piézométrique hyperbolique, si bien que l'écoulement est non uniforme et le débit unitaire croissant: c'est le type observé dans les zones de décharge de la nappe, c'est-à-dire dans les vallées de la Sota et du Niger ; (iii) le type à module d'espacement constant, traduisant un profil piézométrique linéaire, si bien que l'écoulement est uniforme et le débit unitaire constant: c'est le type observé sur les flancs des dépressions localisées entre les deux zones précédentes, c'est-à-dire dans les zones d'écoulement entre les lieux de recharge et ceux de drainage de la nappe. 


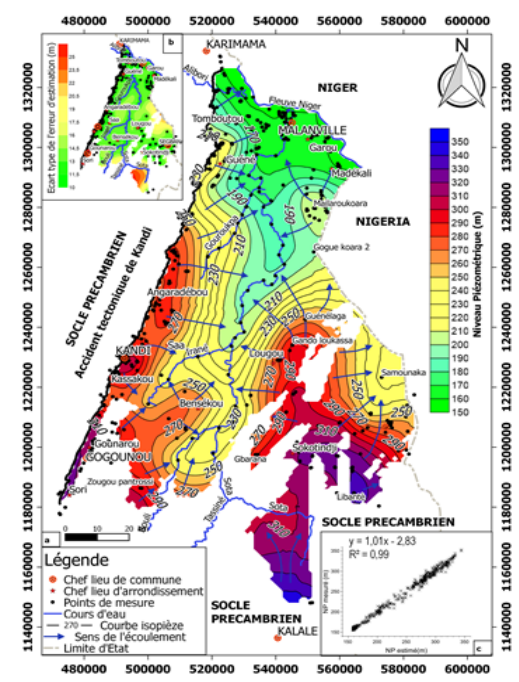

Figure 13. Carte piézométrique de la nappe du bassin sédimentaire de Kandi (août 2016, période des hautes eaux)

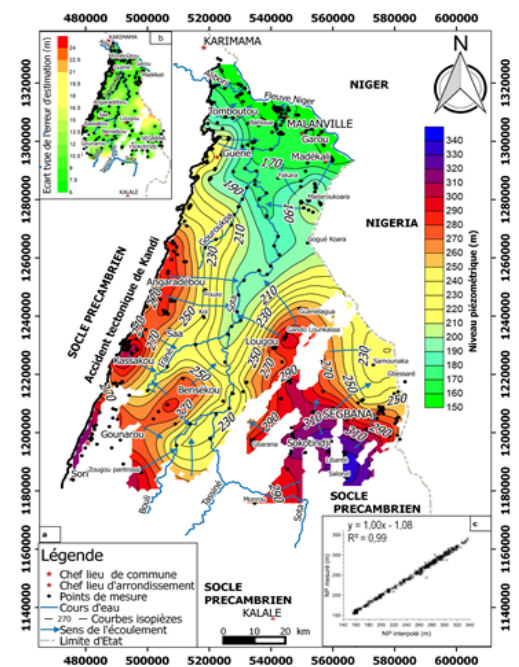

Figure 14. Carte piézométrique de la nappe phréatique du bassin sédimentaire de Kandi (avril 2017, période des basses eaux)

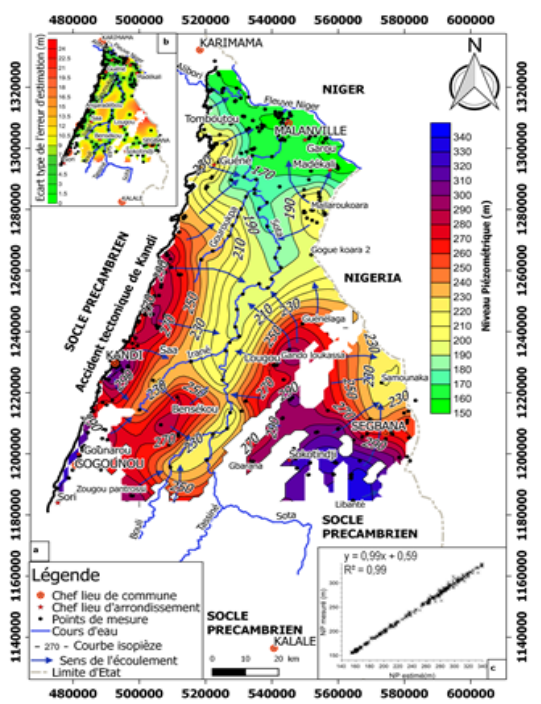

Figure 15. Carte d'isovaleurs des moyennes de niveaux piézométriques mesurés durant la période des hautes eaux (août 2016) et ceux mesurés durant la période des basses eaux (avril 2017) du bassin sédimentaire de Kandi (période des moyennes eaux)

Enfin, il faut préciser que le grand écartement des isopièzes (faible gradient hydraulique) vers la vallée du Niger et le cours aval de la Sota, traduirait une augmentation locale de la perméabilité de l'aquifère et/ou un aplanissement géomorphologique du terrain (Alassane, 2004). 


\section{Synthèse et discussion}

Plusieurs formations hydrogéologiques composent le BSK qui sont de bas en haut, la Fomation de Wèrè, la Formation de Kandi, la Formation de Sendé, la Formation siderolithique dite du Continental Terminal et les alluvions du Quaternaire. Mais seules les deux premières couvrent l'entièreté, ou presque, du bassin. La FS et le CT sont peu épais, latéralement peu étendus et hydrogéologiquement dénoyés, donc stériles. Le Quaternaire est également peu épais et ne semble être saturé que sur une faible épaisseur. Quant à la Formation de Wèrè, même si elle constitue l'essentiel du remplissage du BSK, elle n'est captée directement par les forages d'exploitation existants que sur ses bordures ouest et sud où elle affleure. C'est donc la nappe de la formation supérieure dite de Kandi qui semble être fréquemment exploitée. Toutefois, il n'est pas exclu que certains au moins de ces forages traversent entièrement cette formation, dont l'épaisseur n'est en moyenne que de $75 \mathrm{~m}$, pour aller capter en fait le sommet de la Formation de Wèrè. Dans tous les cas, il est difficile de faire la part entre les nappes des deux formations, celles-ci étant, semble-t-il, en interconnexion, voire en continuité et en équilibre hydraulique, même si les réservoirs sont rendus hétérogènes aussi bien par des variations de faciès que par des discontinuités d'origine tectonique. Tout se passerait donc comme s'il s'agissait d'un seul et même aquifère. Dans ce contexte, seules des études chimiques et/ou isotopiques, qui sont en cours, pourraient contribuer à faire la part entre les deux eaux souterraines.

L'évaluation des paramètres hydrodynamiques de l'aquifère a été esquissée avec une quantité très limitée de données de pompages d'essai de courtes et longues durées. Les valeurs de transmissivité obtenues avec les données de pompage de courtes durées sont relativement faibles, ce qui n'est pas surprenant quand on sait que de faibles épaisseurs d'aquifère sont crépinées pour l'hydraulique villageoise. Les valeurs de paramètres hydrauliques calculés avec les données d'essais de longues durées devraient être plus proches de la réalité, même si elles demandent à être densifiées et spatialement bien réparties pour être véritablement représentatives du système aquifère étudié, système qui, du reste, ne peut pas être considéré comme homogène aussi bien verticalement que latéralement.

La fiabilité des cartes de profondeur de la nappe et piézométriques établies dépend de la précision des mesures de terrain, mais aussi de la validité des interpolations effectuées, les points de mesures n'ayant pas pu être tous nivelés directement sur le terrain. En outre, une vérification, par des mesures directes sur le terrain, dès que l'accès sera possible, des niveaux piézométriques estimés sur la portion amont du fleuve Sota et ses affluents avec la source d'altitude (CGIAR-CSI v4.1 90m x 90m) retenue pour les calculs des altitudes, est nécessaire. De ce point de vue, les résultats de l'étude des échanges entre la nappe phréatique et les cours d'eau du bassin par les 
méthodes chimiques et isotopiques en cours seront utiles. Malgré toutes les réserves émises ci-dessus, les cartes piézométriques élaborées dans le cadre de la présente étude devraient être plus fiables que celles, antérieures, de Turkpak International-SCET-Tunisie (1991) et de Kpegli et al. (2015), en raison de la densité, de loin plus élevée, des sites de mesures et de l'approche d'interpolation des données par krigeage adoptée, approche statistique qui offre en effet une possibilité de contrôle des résultats des calculs. Par ailleurs, le drainage par le fleuve Niger de l'aquifère superficiel de la portion béninoise du grand bassin de l'Iullemeden mise en évidence par les cartes produites, avait été également ressorti par la carte piézométrique de la portion du bassin située sur la rive gauche du fleuve, établie par OSS en 2008. Ce drainage de l'aquifère par les eaux de surface s'observe aussi au niveau du bassin du lac Tchad où le lac et ses tributaires sont alimentés par l'aquifère sous-jacent comme le montre la carte piézométrique réalisée par BGR en 2009.

Aux fins d'implantation des forages d'exploitation à partir des cartes piézométriques établies, trois types hydrodynamiques peuvent être pris en considération selon la classification de Castany (1982), utilisée par Gbewezoun (2017): (i) le type favorable à l'implantation de forages d'exploitation, correspondant aux secteurs de décharge de la nappe (vallée du Niger et partie aval de la vallée de la Sota notamment); (ii) le type moyennement favorable, correspondant aux flancs des dépressions, donc les zones d'écoulement entre les zones de recharge et celles de drainage de la nappe ; (iii) le type peu favorable à défavorable à l'implantation des forages, correspondant aux secteurs de recharge de la nappe, c'est-à-dire les extrémités ouest et sud du bassin; en effet, ces zones de recharge peuvent se vider partiellement, voire s'assécher durant la période d'étiage.

\section{Perspectives}

Les paramètres hydrodynamiques du BSK évalués, qui restent à être densifiés et bien distribués spatialement, joints aux cartes de profondeur de la nappe et piézométriques établies, peuvent orienter l'implantation des ouvrages d'approvisionnement en eau potable des populations du bassin. Associées à une future carte de la puissance de l'aquifère, ces informations peuvent servir de base à des projets ultérieurs de modélisation hydrodynamique du système hydrogéologique étudié. Au total, tous ces résultats constituent un puissant support d'aide à la décision pour les autorités du secteur de l'eau au Bénin et, de ce fait, contribuent notablement à l'atteinte des Objectifs de Développement Durable (ODD) de l'Organisation des Nations Unies dans le sous-secteur de l'approvisionnement en eau potable. 


\section{Conclusion}

La présente étude apporte un éclairage sur l’hydrogéologie de la partie superficielle du système aquifère du BSK, notamment sur la nappe phréatique exploité par les forages d'hydraulique villageoise existants sur le terrain. Les résultats montrent que, des cinq formations géologiques constitutives de BSK, seules les deux de base (Formation de Wèrè et Formation de Kandi) servent de réservoirs aux eaux souterraines. Les trois formations les plus récentes (Formation de Sendé, Formation du Continental Terminal et Formation alluviale du Quaternaire) sont pour l'essentiel dénoyées ou presque, donc stériles ou quasi-stériles.

Les valeurs de transmissivité de cet aquifère superficiel obtenues sont, dans l'ensemble, de l'ordre de $10^{-4}$ s'agissant des pompages d'essai de courte durée, et de l'ordre de $10^{-2}$ s'agissant des pompages de longues durées. Quant aux valeurs de coefficients de perméabilité, elles sont de l'ordre de $10^{-5}$ dans l'ensemble s'agissant des pompages d'essai de courtes durées, et de $10^{-4}$ à $10^{-3}$ s'agissant des pompages de longues durées. Les valeurs des paramètres hydrauliques calculés avec les données d'essais de longues durées sont susceptibles d’être plus proches de la réalité, mais elles nécessitent d’être densifiées et spatialement bien réparties pour être véritablement représentatives du système aquifère étudié, système qui présente, en effet, une certaine hétérogénéité liée aux variations de faciès et aux discontinuités tectoniques.

Les cartes de profondeur de la nappe dressées pour la nappe phréatique objet de la présente étude, montrent que les NS varient de 0 à environ $40 \mathrm{~m}$, les deux saisons confondues. En période des hautes eaux, les niveaux statiques les plus faibles, inférieurs à $10 \mathrm{~m}$ notamment, se rencontrent fréquemment dans la moitié sud du bassin et, de façon systématique, sur ses bordures ouest d'une part, nord (dans les environs du fleuve Niger) d'autre part. Les NS moyens, compris entre 10 et $20 \mathrm{~m}$, se retrouvent dans la zone centrale du bassin. Les NS les plus élevés, supérieurs à $20 \mathrm{~m}$, caractérisent, essentiellement, une petite bande de terrain orientée NNW-SSE à l'extrême nord-est du bassin, qui s'étend du Sud de la localité de Tomboutou à celle de Gogué Koara 2. En période des basses eaux, la même distribution spatiale des NS s'observe, à la différence que les zones des faibles valeurs, inférieures à $10 \mathrm{~m}$, sont drastiquement réduites, si bien que les NS, qui ont sensiblement augmenté, sont supérieurs à $10 \mathrm{~m}$ dans la quasi-totalité de l’étendue du bassin.

Les cartes piézométriques établies montrent que l’écoulement de la nappe phréatique étudiée tend à s’opérer, de façon générale, du Sud vers le Nord, conformément au pendage régional des couches. Mais, les dépressions liées au réseau hydrographique de la Sota et ses affluents et la structuration tectonique du bassin ayant contrôlé étroitement les directions des apports sédimentaires, font que cet écoulement échappe au contrôle strict par le 
pendage régional nord à NNE des couches géologiques. Ainsi, on distingue des zones de recharge de la nappe, localisées sur les bordures sud et ouest du bassin, et deux zones de drainage, l'une, à fort gradient hydraulique (4,3 \%o) et interne au bassin, correspond aux vallées de la Sota et de ses affluents et l'autre, à faible gradient hydraulique (2,8 \%o), correspond à la bordure nord du bassin, c'est-à-dire à la vallée du fleuve Niger (rive sud) et au cours aval de la Sota. Les NP varient d'environ $310 \mathrm{~m}$ au Sud à $150 \mathrm{~m}$ au Nord pour les hautes eaux et d'environ $300 \mathrm{~m}$ au Sud à $150 \mathrm{~m}$ au Nord pour les basses et moyennes eaux. Le battement de la nappe est donc remarquable au Sud, où il peut atteindre et dépasser une dizaine de mètres, mais il n'est plus que de quelques mètres au Nord dans la zone de décharge de la nappe.

\section{Remerciements}

Ce travail a été réalisé grâce aux données, aux travaux de terrain et aux appuis financiers de sources diverses. Les auteurs remercient la Direction Générale de l'Eau (DG-Eau) et les Services Eau des départements de l'Alibori et du Borgou, la Société Nationale des Eaux du Bénin (SONEB), l'Institut Géographique National du Bénin (IGN Bénin), l’Agence International pour l'Energie Atomique (AIEA), l'Observatoire du Sahara et du Sahel (OSS) et la Banque Islamique de Développement (BID). Ils remercient également les évaluateurs pour leurs disponibilités et leurs contributions.

\section{References:}

1. Achidi, J., Bourguet, L., Elsaesser, R., Legier, A., Paulvé, E. \& Tribouillard, N. (2012). Notice explicative de la carte hydrogéologique du Bénin : carte du bassin sédimentaire de Kandi à l'échelle 1/200 000. Rapport Technique, DG-Eau, Cotonou, Bénin, 45p.

2. Alidou, S. (1983). Etude géologique du basin paléo-mésozoïque de Kandi (Nord-Est du Bénin. Afrique de l'ouest). Thèse Doct. d'Etat, Univ. Nat. du Bénin et Univ. de l’Université de Dijon, France. 328p.

3. Alidou, S. \& Lang, J. (1983). Etude sédimentologique, paléogéographique et stratigraphique du bassin intracratonique paléozoïque-mésozoïque de Kandi (Nord-Est Bénin- Afrique de l’Ouest). Geol. Rundsh, Vol. 72 No. 1, pp. 191-205.

4. Alidou, S. (1987). Etude géologique du bassin paléo-mésozoïque de Kandi Nord-Est du Bénin (Afrique de l’Ouest). Pub. Occas. CIFEG, Paris, No. 13, 119p.

5. Alidou, S., Lang, J., Bonvalot, J., Roman, E. \& Seilacher, A. (1991). Marine influences in the socalled continental sediments of the Paleozoic-Mesozoic Kandi Basin (Northern Benin - West Africa). J. Afr.Earth Sci., Vol. 12, No. 1/2, pp. 55-65. 
6. Azontonde, H. A. (1991). Propriétés physiques et hydrauliques des sols au Bénin. Soil Water Balance in the Sudano-Sahelian Zone (proceedings of the Niamey Workshop). IAHS Publ. No. 199, pp. 249258.

7. BGR (2009) : A review of the groundwater situation in the Lake Chad Basin. Report, 18 p.

8. Bois, Ph., Obled, Ch. \& Zin, I. (2007). Introduction au traitement de données en hydrologie Institut National Polytechnique de Grenoble, ENSHMG. 7ème édition revue et complétée, L’Edition du Millénaire, 265p.

9. Boukari, M., (2007). Hydrogéologie de la République du Bénin (Afrique de l'Ouest). Africa Geoscience Review Vol. 14 No. 3, pp. 303-328.

10. Boukari M. (2012). Projet régional AIEA RAF7011 - Gestion intégrée et durable des systèmes aquifères et bassins partagés des Etats membres de la région du sahel. Document Power-Point, 28p.

11. Castany, G., (1982). Principes et méthodes de l'hydrogéologie. Paris: Dunod, 236p.

12. CEFIGRE ou Centre de Formation Internationale à la Gestion des Ressources en Eau (1984). Synthèse des connaissances sur l'hydrogéologie du socle cristallin et cristallophyllien, et du sédimentaire ancien de l'Afrique de l'Ouest. Rapport Ministère des Relations Extérieures, Valbonne, France. 121p.

13. Gbewezoun, H. G. V. (2013). Caractérisation hydrogéologique de l'aquifère du plateau d'Allada dans la zone de Kpanroun (commune d'Abomey-Calavi). Mémoire de Licence, UAC / FAST / Formation en Hydrologie, 47p + annexes.

14. Gbewezoun, H. G. V. (2017). Caractérisation structurale et hydrodynamique de l'aquifère superficiel du Bassin Sédimentaire de Kandi (Nord-Est du Bénin). Mémoire de Master, UAC/INE/HGRE, $62 p+$ annexes.

15. INSAE ou Institut National de la Statistique et de l'Analyse Economique (2016). Effectifs de la population des villages et quartiers de ville du Bénin (RGPH-4, 2013). 83p.

16. IRB ou Institut de Recherche BREDA (1989). Notice explicative de la carte géologique à 1/200 000 : Feuilles de Kandi et Malanville (1ère édition 1989). Mémoire $\mathrm{n}^{\circ} 2$, Rapport Office Béninois des Mines, 75p.

17. Konaté, M., Guiraud, M., Alidou, S., Clermonté, J., Drouet J.J. \& Lang J. (1994). Structuration et dynamique sédimentaire du bassin paléozoïque en demi-graben de Kandi (Bénin, Niger). Comptes Rendus Académie Sciences, Paris Vol. 318, pp. 535-542. 
18. Konaté, M. (1996). Evolution tectono-sédimentaire du bassin paléozoïque de Kandi (Nord Bénin, Sud Niger). Thèse de Doctorat, Université Nancy I, Université Bourgogne, 302p.

19. Konaté, M., Lang J., Guiraud M., Yahaya M., Denis, M., \& Alidou S. (2006). Un bassin extensif formé pendant la fonte de la calotte glaciaire hirnantienne : le bassin ordovicosilurien de Kandi (Nord Bénin, Sud Niger). Africa Geoscience Review, Vol. 13 No. 2, pp. 157-183.

20. Kpegli, K. A. R., Alassane, A., Trabelsi, R., Zouari, K., Boukari, M., Mama, D., Dovonon, F. L., Yoxi, Y. V., Toro-Espitia, L. E. (2015). Geochemical processes in Kandi Basin, Benin,West Africa: A combined hydrochemistry and stable isotopes approach. Quaternary International, Vol. 369, pp. 99-109.

21. Kruseman, G. P., De Ridder N. A. \& Meilhac, A. (1974). Interprétation et discussion des pompages d'essai. International Institute for Land Reclamation and Improvement, Wageningen, The Netherlands 1974 [Traduction du Bulletin 11 : Analysis and Evaluation of Pumping Test Data ; ILRI Wageningen, 1973, 2nd edition] 196p+annexes.

22. Lang, J., Kogbe, C., Alidou, S., Alzouma K. \& AL. (1986) Le sidérolithique du Tertiaire ouest-africain et le concept de Continental Terminal. Bull. Soc. Géol Fr., Vol. 8 No. 2, pp. 605-622.

23. Lang, J., Kogbe, C., Alidou, S., Alzouma, K. A., Bellion, G., Dubois, D., Durant, A., Guiraud, R., Houessou, A., De Klasz, I., Roman, E., Salardachebodaeef, M. \& Trichet, J. (1990). The continental Terminal in West Africa. J. Afr. Earth Sci, Vol. 10 No. 1/2, pp. 79-99.

24. Lawin, A. E. (2007). Analyse climatologique et statistique du régime pluviométrique de la haute vallée de l'Ouémé à partir des données pluviographiques AMMA-CATCH Bénin. Thèse de Doctorat, Institut National polytechnique de Grenoble, Université d'Abomey-Calavi, 166 p.+annexes.

25. Matheron, G. (1965). Les variables régionalisées et leur estimation. Paris: Masson et Cie, 305p.

26. Moriasi, D., Arnold, J. G., Van Liew, M. W., Bingner, R. L., Harmel, R. D. \& Veith T. L. (2007). Model evaluation guidelines for systematic quantification of accuracy in watershed simulations. Trans. ASABE Vol. 50, pp. 885-900.

27. Nash, J. \& Sutcliffe, J. (1970). River flow forecasting through conceptual models. part i : A discussion of principles. Journal of Hydrology 10, pp.282-290.

28. OSS (Observatoire du Sahara et du Sahel) (2008). The Iullemeden Aquifer System (Mali, Niger, Nigeria). Concerted management of the shared water resources of a Sahelian Transboundary aquifer, Technical Report, Tunis. 
29. Rexer, M. \& Hirt, C. (2014). Comparison of free high-resolution digital elevation data sets (ASTER GDEM2, SRTM v2.1/v4.1) and validation against accurate heights from the Australian National Gravity Database. Australian Journal of Earth Sciences, pp. 1-15, http://dx.doi.org/10.1080/08120099.2014.884983.

30. Seilacher, A. \& Alidou, S. (1988). Ordovician and Silurian trace fossils from Northern Benin (WAfrica). N. Jb. Geol. Paläont. Mh., Stuttgart No. 7, pp. 431-439.

31. SERHAU ou Service d'Etude et de Recherche pour l'Habitat et l’Urbanisme. (1992). Atlas cartographique de la région nord du Bénin. Rapport, MEHU, Cotonou, Bénin, 54p.

32. THEIS C.V. (1935) The relation between the lowering of the piezometrie surface and the rate and duration of discharge of a weil using groundwater storage. Am. geoph. union Trans, 16th ann. meeting, p. 519-524, Washington.

33. Turkpak International-SCET-Tunisie (1991). Inventaire des ressources en eaux souterraines au Bénin. Rapport final, Direction de l’Hydraulique, Cotonou, Bénin. (1). 284p 